\title{
ELEVACIÓN DE UNA CÚPULA DE HORMIGÓN EN EL PALACIO DE CONGRESOS DE SALAMANCA. ESPAÑA
}

\author{
(CONCRETE DOME ELEVATION AT THE CONGRESS AND EXPOSITIONS PALACE OF \\ SALAMANCA. SPAIN)
}

Pablo Díaz-Romeral, Arquitecto ICCET/CSIC

\section{RESUMEN}

Se describe en este artículo el izado de una cúpula rígida, tipo baldaquino, de $1.400 t$ de peso y construida separadamente del resto del edificio del Palacio de Congresos y Exposiciones de Salamanca.

La cúpula, que ocupa una superficie aproximada de $1.100 \mathrm{~m}^{2}$ dentro de un cuadrado de $33 \mathrm{~m}$ de lado, fue izada mediante dieciséis gatos hidráulicos de 84 t da carga cada uno.

El proyecto es obra del Arquitecto Juan Navarro Baldeweg, con el cual obtuvo el primer premio en el Concurso de Proyectos.

\section{SUMMARY}

It's described in this joint the elevation of a rigid dome, kind of baldaquino, with a weight of a 1,400 t, and built separately of the building remainder of the Congress and Expositions Salamanca's Palace.

The dome has an area of $1,100 \mathrm{~m}^{2}$, inside a square of $33 \mathrm{~m}$ each side, it has been hoisted by means of sixteen hidraulic cats of $84 t$ of load power each one.

The author is the architect Juan Navarro Baldeweg who won the first price in a competition.
El proyecto ha sido redactado por el Dr. Arquitecto don Juan Navarro Baldeweg con el criterio de incorporar el nuevo edificio al conjunto monumental de la ciudad de Salamanca, recomponiendo y afirmando la impresión volumétrica simple de la muralla, dando una caracterización como basamento de la ciudad antigua, como construcción mural y como paso y vestíbulo entre el núcleo alto consolidado y el parque de la Vaguada.

El conjunto hace participar tres arquetipos constructivos: el de una arquitectura mural o de caja, el de una arquitectura adintelada y el del baldaquino.

A uno de los lados del acceso peatonal se disponen en una construcción compacta las grandes Salas del Palacio de Congresos. La galería que sobresale al otro lado ofrece una imagen complementaria en la forma de una construcción abierta y articulada como un pequeño templo.

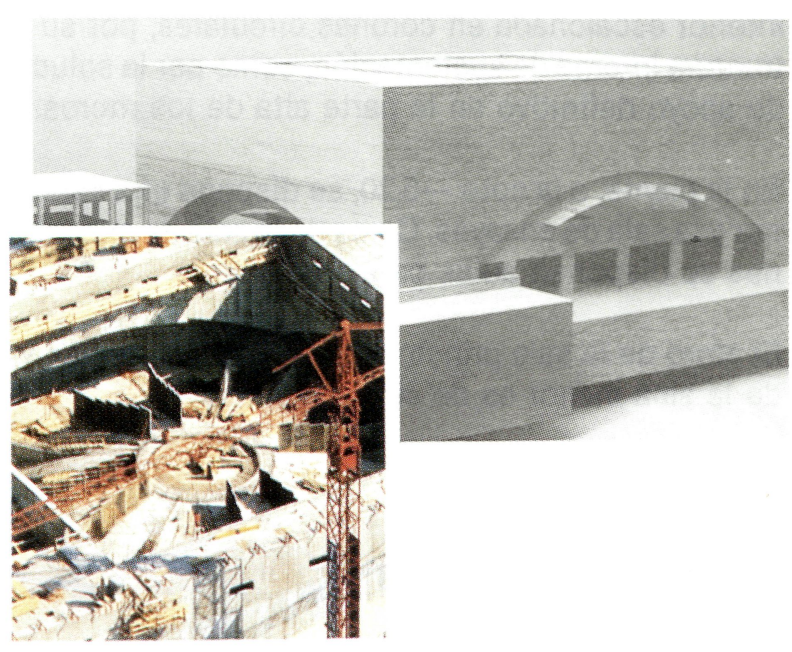

Al acto del izado de la cúpula asistió, en representación del Instituto Eduardo Torroja, el Arquitecto Pablo Díaz-Romeral.

http://informesdelaconstruccion.revistas.csic.es 
La cúpula baldaquino no deja ver en los arcos de penetración al palacio y se dibuja también en los paramentos exteriores del edificio principal.

La cota en la que se implanta el edificio obliga a rechazar cualquier intento de coronación especial que enturbiaría el perfil más alto de la ciudad, incapaz de dialogar adecuadamente, por su inferior posición, con los cuerpos de los monumentos altos.

\section{LA CONSTRUCCIÓN}

El proyecto, tan ambicioso en su diseño arquitectónico, ha estado supeditado totalmente, en su ejecución, por la cúpula baldaquino que es el elemento sobresaliente en el diseño y, a la vez, el que entraña mayores dificultades en la realización de la obra y condiciona todo el proceso constructivo.

El baldaquino está suspendido en los muros de carga pareados que actúan a la vez como grandes vigas que conducen los esfuerzos, por su forma arqueada, a los extremos o vértices del perímetro del edificio.

Por ello, en el proceso constructivo, se eligió el realizar en primer lugar los núcleos de esquina ("pilares"), en toda su altura y, posteriormente, Ilevar a cabo la ejecución de los muros en arco ("vigas") de cierre perimetral del edificio y los interiores que sustentarán la cúpula.

Por tanto, toda la ejecución se ha visto dificultada por las esperas a prever en cada alzado de muros para forjados, vigas, muros en arco, losas curvas prolongación de la cúpula, etc., y por la necesidad de disponer de grandes apuntalamientos en altura y peso.

Por fin, como elemento a ejecutar de mayor dificultad, estaba la propia cúpula baldaquino, tanto por su envergadura, por su forma de casquete esférico con su cara interior escalonada en coronas circulares, por su estructura interna de sustentación, como por la solución de apoyo definitivo en la parte alta de los muros.

En la planta, a la cota $-5,20$, se dispone una sala menor y la sala de ensayos. Cerca de la sala de exposiciones se disponen aulas para seminarios.

El aforo de la sala principal es de 1.300 personas y el de la sala menor es de 460 personas.

\section{LA CÚPULA BALDAQUINO}

El espacio interior del cuerpo principal está concebido como arquitectura dentro de la arquitectura, en la

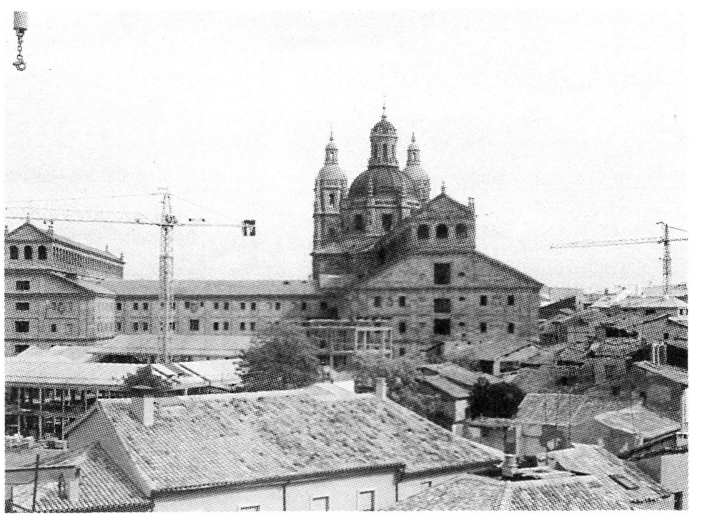

Vista del entorno histórico en el que se sitúa el edificio (Clerecía de Salamanca).

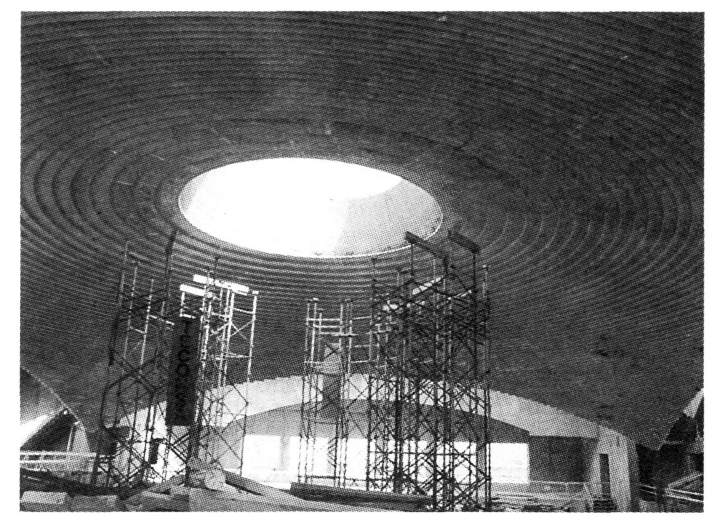

Intradós de la cúpula con relieve regulador de la acústica.

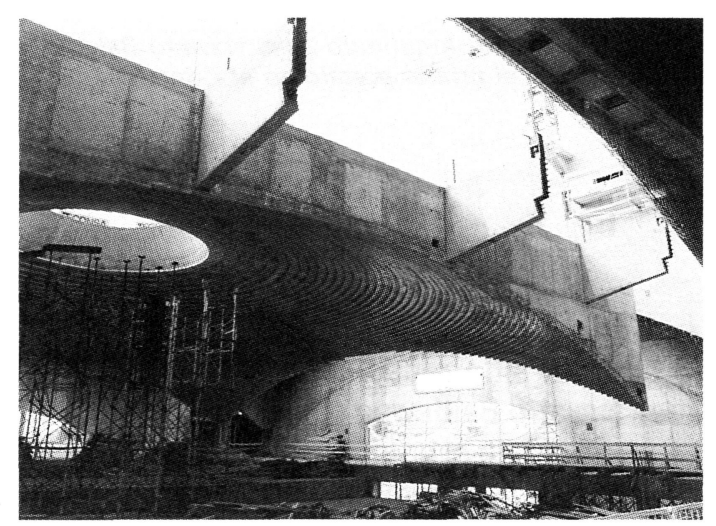

La cúpula en proceso de elevación y ménsulas de ascenso y anclaje al resto de la estructura.

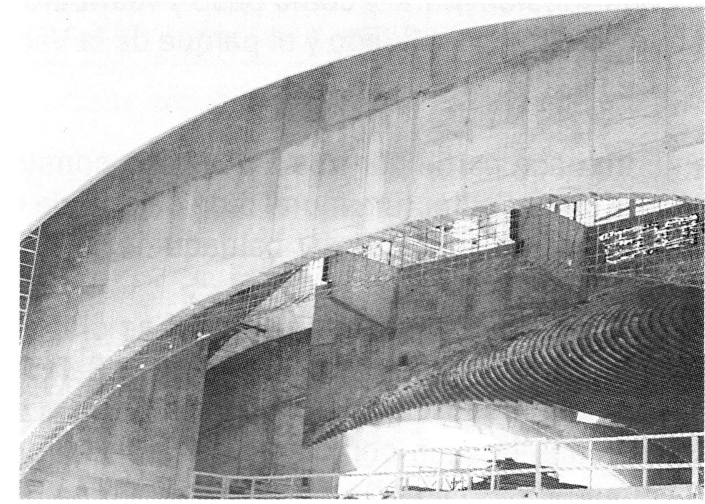

Los “arcos-viga” sobre los que descansará la cúpula. http://informesdelaconstruccion.revistas.csic.es 


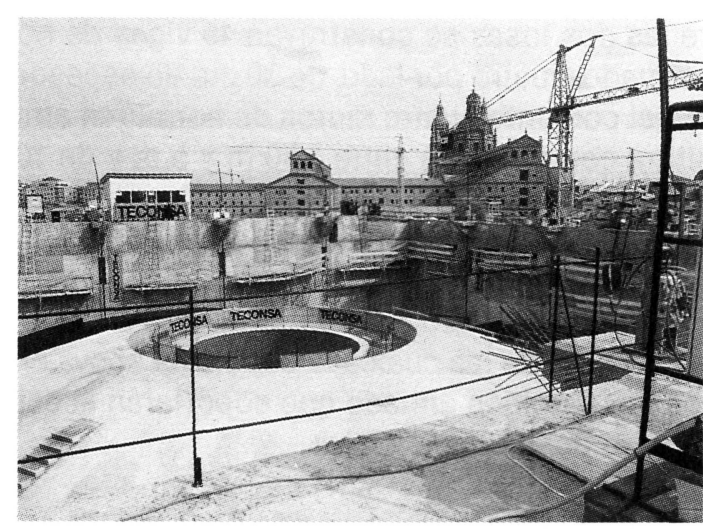

Vista superior de la cúpula. Al fondo los mechinales en espera de las ménsulas.

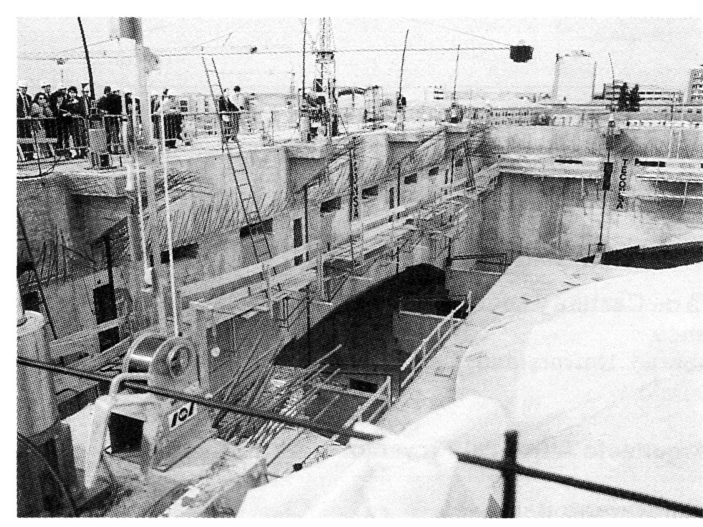

Aspecto general del procedimiento de elevación. Gatos hidráulicos regulados por ordenador estirando de los cables anclados a las mén. sulas.

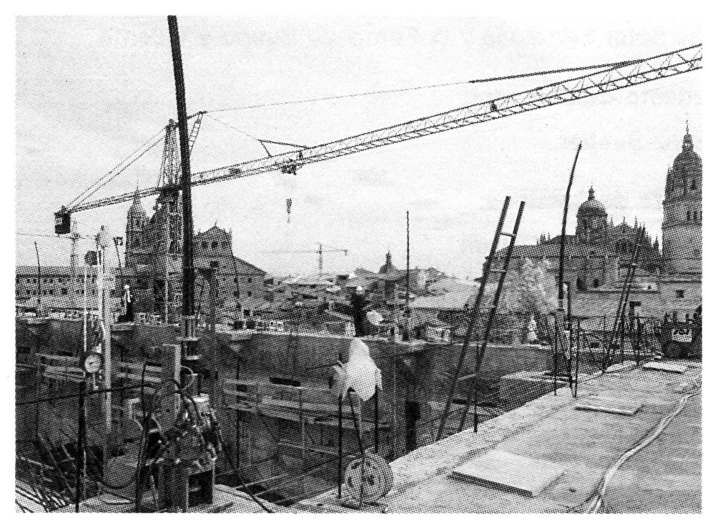

Los gatos hidráulicos proceden a elevar la cúpula.

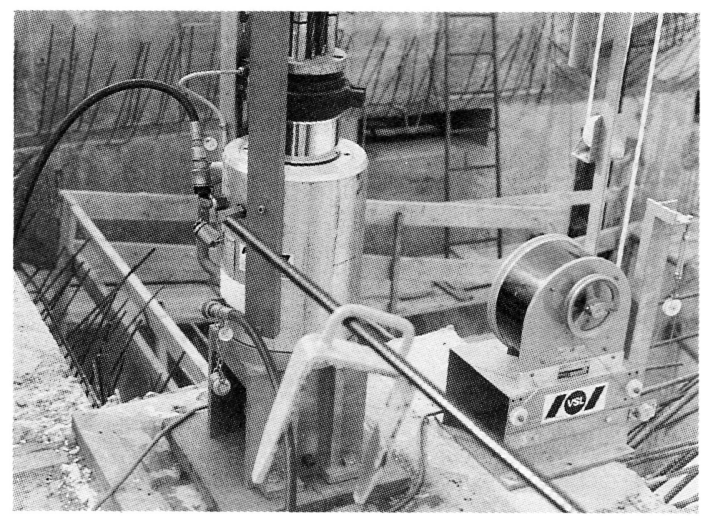

Detalle de la instrumentación empleada. que un gran baldaquino flota sobre el auditorio principal. La percepción de la estructura está reavivada por el tratamiento de la luz natural. La luz cenital dibuja los constituyentes esenciales del espacio interior, conduciendo la vista a las aberturas verticales e iluminando las paredes por donde resbala la luz.

La cara interna de la cúpula está moldeada con un estriado cuya geometría garantiza las exigencias acústicas.

También los arcos pertenecen a la esfera del baldaquino, de manera que no hay ruptura en la perseguida unidad espacial y geométrica.

El baldaquino sobre el auditorio principal, flotando y recortado en la luz, se muestra en una tensión equilibrada entre gravidez e ingravidez.

Planteadas las dificultades de construcción de la cúpula, se adoptó su realización en el suelo de la sala principal y su posterior elevación a la cota definitiva y ello por diversas razones, como son el poder iniciar la construcción de la cúpula en tanto se ejecutaban los muros, la importante reducción del volumen de la cimbra de apeo necesaria y las exigencias técnicas de un desencofrado uniforme en toda la superficie, para la correcta entrada en carga del elemento.

\section{ELEVACIÓN DE LA CÚPULA BALDAQUINO}

Dada la propia estructura de la cúpula, apoyada finalmente en la parte alta de los muros, por medio de dieciséis vigas, distribuidas en todo el perímetro, cuatro en cada cara, el problema de la elevación se simplifi$\mathrm{ca}$, disponiendo una unidad de elevación en cada una de dichas vigas.

Por otro lado, en cubierta, encima de cada viga que sustenta la cúpula, se disponía de una viga para apoyo de la losa inclinada en vuelo sobre el hueco libre entre baldaquino y muros, a la vez que se le podía dar la capacidad de carga necesaria.

Por tanto, el sistema de elevación consiste en situar en las vigas de cubierta, dieciséis gatos hidráulicos de ochenta y cuatro toneladas de cubierta, dieciséis gatos hidráulicos de ochenta y cuatro toneladas de capacidad portante cada uno, que por medio de cables anclados en un elemento metálico dispuesto expresamente en las vigas soporte de la cúpula llevarían a cabo la izada por tracción. 
El conjunto de los gatos hidráulicos están alimentados por varias bombas a través de los correspondientes circuitos hidráulicos, todos ellos controlados mediante un sistema informático centralizado en un cuarto de control.

Por medio de ordenador se controlan en todo momento los niveles alcanzados en los dieciséis puntos de izada, que han de ser iguales. Además hay un control mecánico de altura en diversos puntos, mediante cinta métrica para la lectura directa y sistema de cable con aparato mecánico de lectura.

La cúpula ocupa una superficie en planta de $1.100 \mathrm{~m}^{2}$ aproximadamente, según un cuadrado de $33 \mathrm{~m}$ de lado; su forma es de casquete esférico de $37 \mathrm{~m}$ de radio y su peso es de 1.400 toneladas.

La estructura se construye sobre una losa de hormigón armado de $30 \mathrm{~cm}$ de canto, que constituye el piso de la sala principal. La cúpula está formada por dos losas separadas por una distancia que oscila de $1,50 \mathrm{~m}$ a $5 \mathrm{~m}$; la losa inferior es de hormigón armado con espesor medio de $16 \mathrm{~cm}$ y la superior es un forjado de vigueta pretensada y bovedilla cerámica de $15 \mathrm{~cm}$ de espesor.
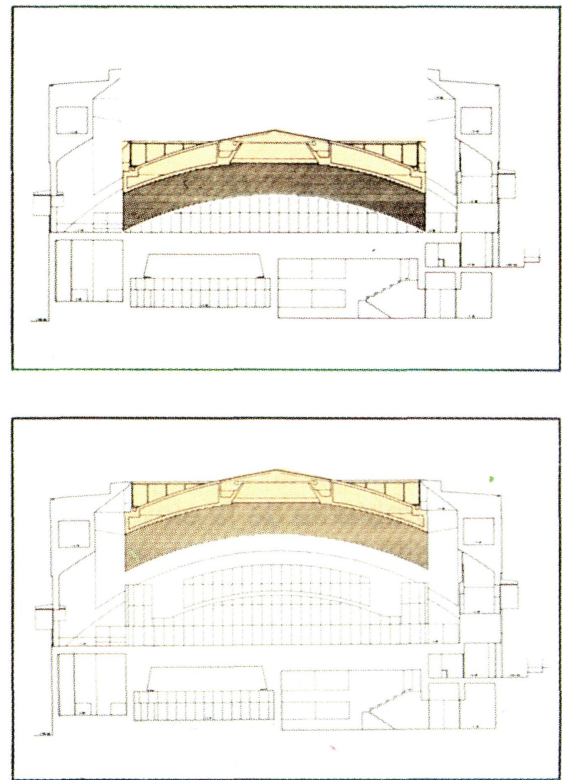

Entre las dos losas se construyen 15 vigas de hormigón armado, cuatro por lado, de $30 \mathrm{~cm}$ de espesor, cerrando el conjunto cuatro muros de hormigón armado de altura comprendida entre $1,50 \mathrm{~m}$ y $5 \mathrm{~m}$ y de $10 \mathrm{~cm}$ de espesor, formando un cuadrado de $30 \mathrm{~m}$ de lado. Prolongando las 16 vigas a partir de los muros de cierre, se construyen 16 vigas mixtas de hormigón armado y acero laminado de $3,25 \mathrm{~m}$ de longitud y $30 \mathrm{~cm}$ de espesor, por medio de las cuales la cúpula se apoya en los muros de hormigón armado que soportarán la estruc. tura en su posición definitiva.

Una vez terminada la construcción de la cúpula se procede a la elevación a su posición definitiva.

PALACIO REGIONAL DE CONGRESOS DE CASTILLA Y LEON EN SALAMANCA

Propiedad:

Patronato del Palacio de Congresos

Junta de Castilla y León, Diputación Provincial, Ayuntamiento de Salamanca.

Colaboran: Universidad, Caja de Ahorros de Salamanca, Cámara de Comercio.

Dr. Arquitecto Autor del Proyecto:

D. Juan Navarro Baldeweg.

Dirección técnica de obra:

Arquitectos:

D. Pau Soler Serratosa y D. Fernando Bueno y Vicente.

Arquitecto Colaborador:

D. Franz Bucher.

Ingeniero de Caminos:

D. Julio Martínez Calzón.

Aparejadores:

D. Eduardo González Velayos, D. José Luis Valverde, D. Juan Chamorro y D. José Luis Castaño.

Ingeniero Técnico:

D. Serafín Portillo.

Empresa Constructora:

TECONSA Tecnología de la Construcción, S. A.

Cl. Serrano, 41-7. ${ }^{\circ}$ - 28001 MADRID 